\title{
Prevalência e fatores associados às malformações congênitas em nascidos vivos
}

Prevalence and factors associated with congenital malformations in live births Prevalencia y factores asociados a las malformaciones congénitas en nacidos vivos

Millena KeIry da Silva Gonçalves ${ }^{1}$ id https://orcid.org/0000-0001-5449-0031

Mirian Domingos Cardoso² id https://orcid.org/0000-0002-2256-8874

Rosário Antunes Fonseca Lima² io https://orcid.org/0000-0002-9110-8329

Conceição Maria de Oliveira ${ }^{3}$ id https://orcid.org/0000-0002-2220-5782

Cristine Vieira do Bonfim ${ }^{1,4}$ io https://orcid.org/0000-0002-4495-9673

Como citar:

Gonçalves MK, Cardoso MD, Lima RA, Oliveira CM, Bonfim CV. Prevalência e fatores associados às malformaçōes congênitas em nascidos vivos. Acta Paul Enferm. 2021;34:eAPE00852.

DOI

http://dx.doi.org/10.37689/actaape/2021A000852

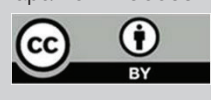

Descritores

Anormalidades congênitas; Estatísticas vitais; Sistemas de Informação em Saúde; Nascido vivo;

Prevalência

Keywords

Congenital abnormalities; Vital statistics; Health information systems; Live birth; Prevalence

Descriptores

Anomalías congénitas; Estadísticas vitales; Sistemas de información en salud; Nacimiento vivo;

Prevalencia

Submetido 10 de Abril de 2019

Aceito

20 de Agosto de 2020

Autor correspondente

Cristine Vieira do Bonfim E-mail: cristine.bonfim@uol.com.br

\section{Resumo}

Objetivo: Estimar a prevalência de malformações congênitas e identificar os fatores associados em nascidos vivos.

Métodos: Estudo transversal, de base populacional, com dados do Sistema de Informações sobre nascidos vivos. Procedeu-se a uma análise estatística bivariada (teste Qui-quadrado) e multivariada (regressão logística múltipla) para avaliar a associação entre as variáveis e o desfecho (nascidos vivos que possuem ou não malformações congênitas).

Resultados: Registraram-se 346.874 nascidos vivos, desses 3.473 apresentaram algum tipo de malformação congênita, com prevalência média de 1,0\%. Na análise múltipla os fatores, positivamente associados à prevalência foram: duração da gestação menor que 37 semanas $(O R=1,17)$, idade materna entre 20 e 29 anos $(\mathrm{OR}=0,893)$, tipo de gravidez única $(\mathrm{OR}=1,775)$, tipo de parto $(\mathrm{OR}=0,827)$ e consultas de pré-natal inferior a seis $(\mathrm{OR}=1,214)$.

Conclusão: As variáveis apontadas no estudo integraram um modelo preditivo que pode auxiliar no planejamento dos serviços de saúde, sugerir hipóteses sobre os fatores etiológicos, e subsidiar as ações do pré-natal com atenção para os fatores identificados.

\section{Abstract}

Objective: To estimate the prevalence of congenital malformations and to identify associated factors in live births.

Methods: Cross-sectional study, population-based, with data from the Live Births Information System. A bivariate statistical analysis (Chi-square test) and a multivariate statistical analysis (multiple logistic regression) were performed to evaluate the association between the variables and the outcome (live-births with or without congenital malformations).

Results: A total of 346,874 live births were registered, of which 3,473 presented some type of congenital malformation, with an average prevalence of $1.0 \%$. In the multiple analysis, the factors positively associated with prevalence were: duration of pregnancy less than 37 weeks $(O R=1.17)$, maternal age between 20 and 29 years $(O R=0.893)$, singleton pregnancy $(O R=1.775)$, type of delivery $(O R-0.827)$, and number of prenatal consultations inferior to six $(\mathrm{OR}=1.214)$.

Conclusion: The variables pointed out in the study integrated a predictive model that can help in the planning of health services, suggest hypotheses regarding etiological factors, and finance prenatal care actions with attention to the identified factors. 


\section{Resumen}

Objetivo: Estimar la prevalencia de malformaciones congénitas e identificar los factores asociados en nacidos vivos.

Métodos: Estudio transversal, de base poblacional, con datos del Sistema de Información sobre nacidos vivos. Se procedió a un análisis estadístico bivariado (prueba $\chi^{2}$ de Pearson) y multivariado (regresión logística múltiple) para evaluar la relación entre las variables y el resultado (nacidos vivos que tienen o no tienen malformaciones congénitas).

Resultados: Se registraron 346.874 nacidos vivos, de los cuales 3.473 presentaron algún tipo de malformación congénita, con prevalencia promedio de 1,0 \%. En el análisis múltiple, los factores asociados de forma positiva a la prevalencia fueron: duración de la gestación menor a 37 semanas (OR=1,17), edad materna entre 20 y 29 años $(O R=0,893)$, tipo de gestación única $(O R=1,775)$, tipo de parto $(O R=0,827)$ y consultas de atención prenatal inferiores a seis $(O R=1,214)$.

Conclusión: Las variables observadas en el estudio integran un modelo predictivo que puede ayudar a la planificación de los servicios de salud, sugerir hipótesis sobre los factores etiológicos y respaldar las acciones de la atención prenatal con énfasis en los factores identificados.

\section{Introdução}

As malformações congênitas são uma causa importante de incapacidade e mortalidade infantil, em especial no período neonatal e constituem um considerável e crescente desafio clínico e de saúde pública, devido ao impacto sobre a saúde da populaçáo. ${ }^{(1,2)}$ No mundo estima-se que 2 a $5 \%$ dos recém-nascidos apresentem alguma malformação e cerca 303.000 morrem no período neonatal. ${ }^{(1,3)} \mathrm{Em}$ muitos países de baixa e média renda, as malformações congênitas não são consideradas uma prioridade de saúde pública e são percebidas pela comunidade médica como eventos raros e não evitáveis. ${ }^{(4)}$

Definem-se malformaçóes congênitas como alteraçôes funcionais e estruturais no desenvolvimento embrionário ou fetal, resultantes de causas genéticas, ambientais ou desconhecidas, que podem ser identificadas no pré-natal, no nascimento, ou, às vezes, mais tarde na infância. ${ }^{(1,5,6)}$ Em sua etiologia, encontram-se as infecçóes (citomegalovirose, rubéola e toxoplasmose), os fatores genéticos (hereditários), os ambientais (condições médicas maternas, abuso de substâncias, infecção, medicamentos, radiação, hipertermia, exposição a produtos químicos, poluição do ar, solventes, pesticidas, metais pesados e anormalidades uterinas). ${ }^{(3,7)}$ Acrescente-se a esses, outros fatores de risco já conhecidos, tais como: idade materna, tabagismo, doenças crônicas, diabetes materna, obesidade e novas ameaças, como a epidemia de Zika em que se tem um cenário propício para o aumento das malformaçôes congênitas.

Outro determinante indireto das malformaçóes congênitas é a baixa renda, em que cerca de $94 \%$ das malformaçóes graves ocorram em países de baixa e média renda. Como determinante indireto, esse maior ris- co está relacionado à possível falta de acesso a alimentos nutritivos e suficientes pelas mulheres grávidas, a uma maior exposição a agentes ou fatores como infecção e álcool, ou a um menor acesso à assistência médica. ${ }^{(1)}$

No Brasil, a prevalência geral não difere daquela encontrada em outras regióes do mundo, alcançando aproximadamente de 2 a $5 \%$ de todos os nascidos vivos. $^{(5,8-11)}$ Recente estudo de caso-controle realizado no país, identificou uma incidência de 3,2\% e apontou como principais fatores de risco a ocorrência de familiares com a mesma malformação congênita, consumo materno de álcool, diabetes gestacional e abortos prévios. ${ }^{(4)}$ Igualmente, ao que foi observado em outros países, a pesquisa conduzida localmente, tem apontado a associação das malformaçóes com a utilização de agrotóxicos. ${ }^{(12)}$ Em adição aos fatores citados, a emergência nacional causada pelo Zika vírus associado à microcefalia aumentou a ocorrência de malformações no Brasil, ${ }^{(13)}$ sendo Pernambuco considerado como o estado epicentro da epidemia, devido ao elevado número de casos. ${ }^{(13)}$

No Estado de Pernambuco, uma pesquisa sobre o perfil epidemiológico dos recém-nascidos com malformação congênita identificou uma proporção de $0,8 \% .^{(14-17)} \mathrm{Na}$ cidade do Recife (PE), as malformaçóes constituem uma das principais causas de mortalidade infantil, em especial no período neonatal. Conhecer a sua prevalência, a natureza da ocorrência e os fatores associados é importante para o diagnóstico precoce, planejamento e alocação de recursos para os serviços de saúde especializados, contribuindo para a melhoria da qualidade de vida e redução da mortalidade infantil. Nessa perspectiva, o objetivo desse estudo foi estimar a prevalência de malformaçóes congênitas e identificar os fatores associados em nascidos vivos no Recife, Pernambuco, 2001 a 2015. 


\section{Métodos}

Trata-se de um estudo epidemiológico observacional do tipo transversal, realizado a partir dos dados dos nascidos vivos com malformaçóes congênitas, de mães residentes no Recife (PE), entre 2001 e 2015. A cidade tinha uma populaçáo de 1.617.183 habitantes em 2015, ${ }^{(18)}$ abrangendo uma extensão territorial de $218.435 \mathrm{~km}^{2}$ e uma densidade demográfica de 7.039,64 habitantes $/ \mathrm{km}^{2}$.

A fonte de dados foi constituída pelo Sistema de Informaçóes sobre Nascidos Vivos (Sinasc). ${ }^{(5)}$ Esse sistema, foi implantado em 1990 e tem como objetivo reunir informaçóes sobre os nascidos no território nacional. ${ }^{(16)}$ Os dados sobre nascidos vivos são coletados a partir da declaração de nascidos vivos (DN). A presença de uma malformação congênita ao nascimento passou a ser registrada na DN a partir do ano de 1999, sendo descrita e codificada segundo a décima revisão da Classificação Estatística Internacional de Doenças e Problemas Relacionados à Saúde (CID-10). No ano de 2011, uma nova versão da $\mathrm{DN}$ foi distribuída pelo país, com alteraçóes nos campos relacionados à coleta de informações sobre malformaçóes congênitas. ${ }^{(17)}$

As variáveis foram selecionadas, dicotomizadas (exceto idade materna), e agrupadas de acordo com as características do recém-nascido: sexo ("feminino" ou "masculino") peso ao nascer ("<2.500 gramas" ou “ $\geq 2.500$ gramas")e índice de Apgar no primeiro (“ $<7$ ” ou " $\geq 7$ ”) e no quinto minutos (“ $<7$ ” ou " $\geq 7$ ”); maternas: idade ("<20 anos" ou " $20-29$ anos" ou " $\geq$ 30 anos"), escolaridade (" $<8$ anos" ou “ $\geq 8$ anos"), gestação: tipo de gravidez ("única” ou "múltipla”), número de consultas no pré-natal ( $<6$ ” ou " $\geq 6$ ") e duração da gestação("<37 semanas" ou $\geq 37$ semanas) e do parto: tipo de parto ("vaginal” ou "cesáreo").

Calculou-se a prevalência de malformaçóes congênitas por ano (número de nascidos vivos com malformação/total de nascidos vivos no mesmo período $\mathrm{x}$ 100) e a taxa prevalência média para o período analisado. A taxa de variação para o valor inicial foi calculada para as variáveis número de nascidos vivos, número de nascidos vivos com malformaçôes congênitas e prevalência de malformação, atendendo à seguinte fórmula: $\Delta \%$ [(Ano final-Ano
inicial)/Ano inicial] $\times 100$, em que, caso o valor dê maior que 1 , houve aumento do indicador para o ano final em relação ao inicial, e caso seja inferior a 1 , uma queda aconteceu. A taxa de prevalência média foi estimada a partir da média amostral. A taxa de crescimento foi calculada utilizando os valores com o menor nível de aproximação possível para obter resultados mais fidedignos.

Realizou-se a análise do preenchimento da variável "detecção de anomalia congênita" e das demais variáveis do estudo antes da análise estatística. $\mathrm{Na}$ sequência, procedeu-se a uma análise estatística bivariada, utilizando o teste Qui-quadrado para identificar associaçóes entre as variáveis do estudo e o evento alvo, que neste caso, é a presença ou não de malformaçóes congênitas nos nascidos vivos. Posteriormente, um modelo de regressão logística múltipla foi ajustado, calculando-se as odds ratio (OR) para cada fator estudado, possibilitando o entendimento de quais podem ser considerados de risco ou proteção.

Os fatores investigados (variáveis independentes) foram: sexo, peso ao nascer, escore de Apgar no $1^{\circ}$ e $5^{\circ}$ minuto de vida, idade e escolaridade materna, tipo de gravidez, número de consulta de pré-natal, duração da gestação e tipo de parto. Todos os cálculos e análises foram realizados utilizando a linguagem $\mathrm{R}$ versão 3.2.2 para Windows e o nível de significância estipulado foi de 5\%.

O projeto do estudo foi aprovado pelo Comitê de Ética em Pesquisas da Fundação Joaquim Nabuco (CAAE: 67399617.6.0000.5619).

\section{Resultados}

Foram notificados 346.874 nascidos vivos no Recife entre 2001 e 2015, dos quais 3.473 apresentaram algum tipo de malformação congênita e 329 (0,1\%) estavam com essa informação sem preenchimento. A prevalência média anual foi de $1,0 \%$, com a máxima de 1,4\% no ano de 2015 e mínima de 0,7\% em 2012. A taxa de variação para o valor inicial da prevalência de nascidos vivos com malformaçóes congênitas foi de $21 \%$ no período considerado, e observou-se aumento de $13,2 \%$ no número de casos de malformação congênita no período (Tabela 1). 
Tabela 1. Distribuição de nascidos vivos e prevalência de malformações congênitas

\begin{tabular}{l|c|c|c}
\hline Ano & $\begin{array}{c}\text { Número de nascidos } \\
\text { vivos }\end{array}$ & $\begin{array}{c}\text { Número nascidos } \\
\text { vivos com MFC }\end{array}$ & $\begin{array}{c}\text { Prevalência nascidos } \\
\text { vivos com MFC (\%) }\end{array}$ \\
\hline 2001 & $25.294^{\mathrm{a}}$ & 296 & 1,2 \\
2002 & $24.307^{\mathrm{b}}$ & 207 & 0,9 \\
2003 & $24.676^{\mathrm{c}}$ & 275 & 1,1 \\
2004 & $22.898^{\mathrm{d}}$ & 250 & 1,1 \\
2005 & $23.207^{\mathrm{e}}$ & 227 & 1,0 \\
2006 & $22.646^{\mathrm{f}}$ & 240 & 1,1 \\
2007 & $22.046^{9}$ & 204 & 0,9 \\
2008 & $22.261^{\mathrm{h}}$ & 184 & 0,8 \\
2009 & $22.546^{\mathrm{i}}$ & 215 & 1,0 \\
2010 & $21.796^{\mathrm{j}}$ & 225 & 1,0 \\
2011 & $22.218^{\mathrm{k}}$ & 178 & 0,8 \\
2012 & $22.633^{\mathrm{l}}$ & 151 & 0,7 \\
2013 & $23.176^{\mathrm{m}}$ & 224 & 1,0 \\
2014 & $23.506^{\mathrm{n}}$ & 262 & 1,1 \\
2015 & $23.664^{\circ}$ & 335 & 1,4 \\
\hline Total & 346.874 & 3.473 & 1,0 \\
$\Delta \%$ & $-6,4$ & 13,2 & 21 \\
\hline
\end{tabular}

MFC - malformação congênita; NV - nascidos vivos. Proporção de nascidos vivos com a informação sobre malformação congênita ignorada: a) $0,18 \%$; b) $0,10 \% ;$ c) $0,09 \%$; d) $0,21 \%$; e) $0,05 \%$; f) $0,07 \%$; g) $0,03 \%$; h) $0,01 \%$; i) $0,08 \%$; j) $0,03 \%$; k) $0,14 \%$; I) $0,15 \%$; m) $0,03 \%$; n) $0,05 \%$; o) $0,08 \%$

Sobre os nascidos vivos com malformaçóes congênitas houve predomínio do sexo masculino $(60,1 \%)$, com peso ao nascer $\geq 2.500 \mathrm{~g}(77,3 \%)$, escores de Apgar satisfatórios no primeiro e no quinto minutos $(68,4 \%$ e $86,6 \%)$. As mães tinham entre 20 e 29 anos de idade $(49,1 \%)$, com oito ou mais anos de estudo (65,3\%), com gravidez do tipo única $(97,5 \%)$, que realizaram menos que seis consultas de pré-natal $(52,9 \%)$, com duração da gestação de 37 ou mais semanas $(78,9 \%)$ e tiveram seus filhos por parto cesáreo $(55,5 \%)$ (Tabela 2 ).

Em relação ao sexo, houve menor prevalência no feminino (OR=0,70; IC 95\%=0,65-0,75). Encontrou-se maior chance de anomalias em prematuros com idade gestacional abaixo de 37 semanas $(\mathrm{OR}=1,17$; IC 95\% 1,04-1,32) e em recém-nascidos com peso ao nascer menor que $2.500 \mathrm{~g}(\mathrm{OR}=2,093$; IC 95\% 1,86-2,36) e Apgar no $1^{\circ}(\mathrm{OR}=1,825$; IC $95 \% 1,67-2,00)$ e no $5^{\circ}$ minuto $(\mathrm{OR}=3$ IC $95 \%$ 2,64-3,41) insatisfatório. Evidenciou-se associação entre gestaçôes únicas $(\mathrm{OR}=1,775$; IC 95\% 1,41$2,23)$ e tipo de parto vaginal $(\mathrm{OR}=0,827$; IC $95 \%$ 0,77-0,89). Houve associação dos casos de malformação congênita naquelas mães que realizaram menos que seis consultas de pré-natal $(\mathrm{OR}=1,214$; IC95\% 1,13-1,31) (Tabela 3).
Tabela 2. Características do recém-nascido, maternas, da gravidez e do parto, segundo a presença de malformação congênita

\begin{tabular}{|c|c|c|c|}
\hline \multirow{3}{*}{ Características } & \multicolumn{2}{|c|}{ Malformação } & \multirow{3}{*}{$p$-value } \\
\hline & Sim & Não & \\
\hline & $n(\%)$ & $n(\%)$ & \\
\hline Sexo ${ }^{a}$ & & & $<0,001^{*}$ \\
\hline Feminino & $1310(39,9)$ & $160296(48,9)$ & \\
\hline Masculino & $1974(60,1)$ & $167694(51,1)$ & \\
\hline Peso ao nascer (gramas) ${ }^{b}$ & & & $<0,001^{*}$ \\
\hline$<2.500$ & $745(22,7)$ & $28204(8,6)$ & \\
\hline$\geq 2.500$ & $2539(77,3)$ & 299786(91,4) & \\
\hline Apgar $1^{\circ}$ minuto $0^{\circ}$ & & & $<0,001^{*}$ \\
\hline$<7$ & $1039(31,6)$ & $44221(13,5)$ & \\
\hline$\geq 7$ & $2245(68,4)$ & $283769(86,5)$ & \\
\hline Apgar $5^{\circ}$ minuto ${ }^{d}$ & & & $<0,001^{*}$ \\
\hline$<7$ & $439(13,4)$ & $7257(2,2)$ & \\
\hline$\geq 7$ & $2845(86,6)$ & $320733(97,8)$ & \\
\hline $\begin{array}{l}\text { Idade da mãe (em anos } \\
\text { completos) }\end{array}$ & & & $0,003^{*}$ \\
\hline$<20$ & $663(20,2)$ & $62962(19,2)$ & \\
\hline $20-29$ & $1611(46,0)$ & $170666(52,0)$ & \\
\hline$\geq 30$ & $1010(30,8)$ & $9436(28,8)$ & \\
\hline Escolaridade $(\text { anos })^{f}$ & & & $0,011^{*}$ \\
\hline$<8$ anos & $1140(34,7)$ & $106991(32,6)$ & \\
\hline$\geq 8$ anos & $2144(65,3)$ & $220999(67,4)$ & \\
\hline Tipo de gravidez $z^{9}$ & & & 0,085 \\
\hline Única & $3201(97,5)$ & $321167(97,9)$ & \\
\hline Múltipla & $83(2,5)$ & $6823(2,1)$ & \\
\hline Consulta pré-natal ${ }^{\text {h }}$ & & & $<0,001^{*}$ \\
\hline$<6$ & $1736(52,9)$ & $146588(44,7)$ & \\
\hline$\geq 6$ & $1548(47,1)$ & $181402(55,3)$ & \\
\hline $\begin{array}{l}\text { Duração da gestação (em } \\
\text { semanas)i }\end{array}$ & & & $<0,001^{*}$ \\
\hline$<37$ & $693(21,1)$ & $29950(9,1)$ & \\
\hline$\geq 37$ & $2591(78,9)$ & $298040(90,9)$ & \\
\hline Tipo de partol & & & $0,001^{\star}$ \\
\hline Vaginal & $1459(44,4)$ & $155571(47,4)$ & \\
\hline Cesáreo & $1825(55,5)$ & $172419(52,6)$ & \\
\hline
\end{tabular}

*Rejeitou-se a Hipótese Nula, pois o $p$-value é menor do que o nível de significância de 0.05 (5\%). Proporção dos nascidos vivos com a informação ignorada: a) $0,04 \%$; b) $0,02 \%$; c) $0,8 \%$; d) $0,5 \%$; e) $0,06 \%$; f) $1,2 \%$; g) $0,04 \%$; h) $1,4 \%$; i) $0,5 \%$; j) $0,05 \%$

\section{Discussão}

Os resultados do estudo evidenciaram uma redução no número de nascidos vivos, porém houve aumento na ocorrência e na proporção de nascidos vivos com malformação congênita. Estimouse uma prevalência média de malformaçôes de 1,0 caso para cada 100 nascidos vivos. A análise múltipla teve como fatores positivamente associados à prevalência: baixo peso ao nascer, escore de Apgar insatisfatório no $1^{\circ}$ e $5^{\circ}$ minuto de vida, gravidez do tipo única, número de consultas de pré-natal inferior a seis, prematuridade $(<37$ semanas) e tipo de parto. Essas informações são re- 
Tabela 3. Odds ratio, intervalo de confiança e significância ajustada pela regressão logística múltipla, segundo variáveis que descrevem as características do recém-nascido, maternas, da gravidez e do parto

\begin{tabular}{|c|c|c|c|}
\hline Variável & $\begin{array}{l}\text { Odds ratio } \\
\text { (ajustado) }\end{array}$ & $\begin{array}{c}\text { Intervalo de } \\
\text { confiança (95\%) }\end{array}$ & $p$-value \\
\hline Sexo & & & - \\
\hline Feminino & 0,701 & $(0,65-0,75)$ & $<0,001^{*}$ \\
\hline Masculino & 1 & - & - \\
\hline \multicolumn{4}{|c|}{ Peso ao nascer (gramas) } \\
\hline$<2.500$ & 2,093 & $(1,86-2,36)$ & $<0,001^{*}$ \\
\hline$\geq 2.500$ & 1 & - & - \\
\hline \multicolumn{4}{|c|}{ Apgar $1^{\circ}$ minuto } \\
\hline$<7$ & 1,825 & $(1,67-2,00)$ & $<0,001^{\star}$ \\
\hline$\geq 7$ & 1 & - & - \\
\hline \multicolumn{4}{|c|}{ Apgar $5^{\circ}$ minuto } \\
\hline$<7$ & 3 & $(2,64-3,41)$ & $<0,001^{*}$ \\
\hline$\geq 7$ & 1 & - & - \\
\hline \multicolumn{4}{|c|}{ Idade da mãe (em anos completos) } \\
\hline$<20$ & 0,92 & $(0,83-1,02)$ & 0,115 \\
\hline $20-29$ & 0,893 & $(0,82-0,97)$ & $0,006^{*}$ \\
\hline$\geq 30$ & 1 & - & - \\
\hline \multicolumn{4}{|c|}{ Escolaridade (anos) } \\
\hline$<8$ anos & 1,055 & $(0,98-1,14)$ & 0,181 \\
\hline$\geq 8$ anos & 1 & - & - \\
\hline \multicolumn{4}{|c|}{ Tipo de gravidez } \\
\hline Única & 1,775 & $(1,41-2,23)$ & $<0,001^{*}$ \\
\hline Múltipla & 1 & - & - \\
\hline \multicolumn{4}{|c|}{ Consulta pré-natal } \\
\hline$<6$ & 1,214 & $(1,13-1,31)$ & $<0,001^{\star}$ \\
\hline$\geq 6$ & 1 & - & \\
\hline \multicolumn{4}{|c|}{ Duração da gestação (em semanas) } \\
\hline$<37$ & 1,17 & $(1,04-1,32)$ & $0,012^{*}$ \\
\hline$\geq 37$ & 1 & - & - \\
\hline \multicolumn{4}{|l|}{ Tipo de parto } \\
\hline Vaginal & 0,827 & $(0,77-0,89)$ & $<0,001^{\star}$ \\
\hline Cesáreo & 1 & - & - \\
\hline
\end{tabular}

levantes para a vigilância, prevenção e melhoria nos cuidados especializados.

De início, cabe uma análise sobre a qualidade da base de dados do Sinasc utilizada nesse estudo. A qualidade das informaçóes sobre nascidos vivos são indispensáveis para a vigilância das malformaçóes. A ausência de preenchimento da variável "detecção de malformação congênita" foi de $0,1 \%$. Igualmente, para as demais variáveis o percentual de incompletude (ignorados + em branco) foi considerado excelente. Os maiores percentuais foram para as variáveis escolaridade materna e número de consultas de pré-natal, com percentual inferior a $1,5 \%$. O Sinasc é um sistema com cobertura acima de 90\% na maior parte das unidades da federação, fidedigno $^{(16)}$ e com elevada completude das variáveis. (19) De maneira semelhante, os estudos realizando no estado de Pernambuco e na cidade do Recife tem demonstrado melhoria na adequação das informaçôes do Sinasc. ${ }^{(5,20)}$

Especificamente para a variável "detecção de anomalia congênita" pesquisa que avaliou os atributos e a utilidade do Sinasc no Brasil, mostrou percentual de informação ignorada de 2,5. A análise dessa mesma variável feita para o Recife verificou 99,9\% de preenchimento. ${ }^{(5)}$ Cosme et al. ${ }^{(10)}$ afirmam que o preenchimento correto dessa variável permite examinar a frequência das malformaçôes congênitas, a fim de criar indicadores confiáveis de saúde, aplicáveis ao planejamento de políticas de saúde infantil. Dados de alta qualidade são essenciais para gerar informações para açôes de saúde pública e para a vigilância das malformaçôes congênitas. ${ }^{(21,22)}$

Estimou-se uma prevalência média de 1,0 caso para cada 100 nascidos vivos (1,0\%). A prevalência encontrada no estudo assemelha-se as de outras cidades e estados do país. Na cidade de Salvador (BA), a prevalência foi de 1,0\%, 1,6\% em São Paulo (SP), 0,9\% no estado do Rio Grande do Sul e 0,58\% no Rio Grande do Norte. ${ }^{(10,11,23)}$ Essas variaçóes podem ser em decorrência de diferenças sazonais, ambientais, regionais, ou a falta de detecção de alguns casos de malformações. ${ }^{(24)}$ As causas das malformações são complexas e incluem vários fatores de risco, como idade materna, exposição durante a gestação, localização geográfica, raça/cor e etnicidades. ${ }^{(3)}$

Neste estudo, a maior prevalência de nascidos vivos com malformaçóes ocorreu no ano de 2015. Possivelmente, esses resultados relacionam-se com o acréscimo no número de neonatos com microcefalia ocorrido no Brasil, que se tornou uma epidemia e fez com que o Ministério da Saúde declarasse uma situação de emergência nacional de saúde pública. (25) No estado de Pernambuco, observou-se aumento de nascidos vivos com microcefalia relacionados ao Zika vírus e a maior concentração de casos foi na cidade do Recife. ${ }^{(26)}$ A identificação desses casos demonstra a importância do Sinasc para monitoramento das malformaçóes congênitas e a necessidade do aperfeiçoamento do sistema de vigilância das malformaçóes congênitas. ${ }^{(27)}$

Com relação ao modelo de regressão logística múltipla as variáveis que tiveram associação com o 
risco de desenvolver algum tipo de malformação congênita foram: baixo peso ao nascer $(<2500 \mathrm{~g})$ e escore de Apgar no $1^{\circ}$ e $5^{\circ}$ minuto de vida, idade materna, tipo de gravidez, número de consultas de pré-natal, prematuridade ( $<37$ semanas) e tipo de parto.

Prematuridade, baixo peso ao nascer e índice de Apgar insatisfatório foram associados às malformaçôes congênitas no estudo. Reconhecidamente, existe associação das malformações com restrição de crescimento intrauterino e baixo peso ao nascer. ${ }^{(28,29)}$ A pesquisa que analisou o peso ao nascer por idade gestacional e malformaçóes congênitas, identificou a paridade, o baixo peso ao nascer, a idade gestacional menor que 35 semanas, sexo masculino e falta de pré-natal como fatores de risco mais significativos para malformaçóes congênitas. ${ }^{(30)}$ Nhoncanse et al. ${ }^{(31)}$ apontam que prematuridade, baixo peso e índice de Apgar baixos no $1^{\circ}$ e no $5^{\circ}$ nos nascidos vivos com malformaçóes contribuem para morbidade e mortalidade infantil.

Nesse estudo, idade materna entre 20 e 29 anos, foi a mais frequente e mostrou associação, porém, apresentando menores chances de malformaçóes do que as outras faixas etárias. Há conhecida associação entre idade materna avançada e risco substancialmente mais alto de anormalidades cromossômicas e aborto, potencialmente causadas pelo processo de envelhecimento nos ovários, aumentando a taxa de não disjunção meiótica. ${ }^{(29)}$ Mulheres em idade materna avançada apresentam maiores taxas de incidência de comorbidades (diabetes gestacional, hipertensão induzida pela gravidez, entre outras) e os seus neonatos maiores chances de malformaçóes congênitas. ${ }^{(10,32)} \mathrm{A}$ identificação de mulheres grávidas com maior risco de sofrer um resultado adverso da gravidez, possibilita uma maior vigilância, acompanhamento clínico e assistência pré-natal direcionada.

A realização de menos de seis consultas de pré-natal foi associada às malformaçóes congênitas nos nascidos vivos. Outros estudos também apontaram o menor número de consultas pré-natais como fator associado ao nascimento com malformaçôes congênitas. ${ }^{(30,33)}$ A organização de boas consultas pré-natal, o fortalecimento da atenção primária à saúde e a suplementação com ácido fólico são medidas profiláticas simples, porém eficazes, de prevenção para malformações congênitas. ${ }^{(34)} \mathrm{O}$ início do acompanhamento pré-natal ainda nas primeiras semanas de gestação e a realização do número de consultas preconizadas permite detectar precocemente alteraçóes no feto, bem como previne a exposição a fatores ambientais e teratogênicos. ${ }^{(35)}$

Evidenciou-se a associação estatística com o tipo de gravidez única, porém tem-se observado o predomínio de casos de malformaçóes congênitas em gestações múltiplas. ${ }^{(10)} \mathrm{O}$ maior número de casos das deformidades em gestaçóes múltiplas pode ser explicado, em parte, por erros nas divisóes celulares (fatores genéticos), cromossomopatias, fatores ambientais intraútero e constrição da bolsa amniótica ou de cordão umbilical. ${ }^{(10)}$

Algumas limitações devem ser consideradas ao interpretar os resultados do estudo. A natureza transversal impede o desenho de qualquer relação de causa e efeito entre as malformaçóes e as variáveis analisadas. A utilização de dados secundários que podem estar subnotificados, apresentar erros de classificação e incompletude de informações. Todavia a análise do preenchimento mostrou baixa proporção de incompletude. Para detecção dos defeitos congênitos, um exame clínico e físico sistemático completo e cuidadoso realizado pelo pediatra no nascimento é necessário e deve ser mais enfatizado. Os resultados justificam a relevância do estudo ao fornecer uma imagem representativa da carga de defeitos congênitos entre os nascidos vivos e a análise múltipla permitiu uma inferência sobre as variáveis associadas às malformaçóes congênitas.

\section{Conclusão}

O modelo final de explicação para as malformações congênitas nos nascidos vivos no Recife foi composto por: prematuridade, baixo peso ao nascer, Apgrar no $1^{\circ}$ e $5^{\circ}$ e menor que sete, gravidez única, parto vaginal e menos de seis consultas de pré-natal realizadas. $\mathrm{O}$ conhecimento sobre a prevalência e os fatores associados às malformaçóes congênitas, podem contribuir para o planejamento de ações de saúde materno e infantil, aprimoramento dos métodos de diagnóstico, acompanhamento especializado 
com medidas que melhorem a qualidade de vida, aumentem a sobrevida e a redução da mortalidade infantil no Recife. A elaboração de estratégias de prevenção, detecção precoce e tratamento das malformaçóes congênitas são fundamentais para a redução da morbimortalidade infantil.

\section{Agradecimentos}

Pesquisa financiada pelo Programa Institucional de Bolsas de Iniciação Científica do Conselho Nacional de Desenvolvimento Científico e Tecnológico (Pibic/CNPq) - Processo no 115910/2016-6

\section{Colaborações}

Gonçalves MKS e Bonfim CV contribuíram com a concepção do estudo, análise e interpretação dos dados e redação do manuscrito e aprovam a versão final a ser publicada. Cardoso MD e Lima RAF contribuíram com a revisão crítica de seu conteúdo intelectual e aprovam a versão final a ser publicada. Oliveira CM contribuiu com a análise e interpretação dos dados, redação do manuscrito e aprova a versão final a ser publicada.

\section{Referências}

1. World Health Organization. Congenital anomalies, 2016. Available at: https:// www.who.int/news-room/fact-sheets/detail/congenital-anomalies

2. Feldkamp ML, Carey JC, Byrne JL, Krikov S, Botto LD. Etiology and clinical presentation of birth defects: population based study. BMJ. 2017 May;357(2249):j2249.

3. Harris BS, Bishop KC, Kemeny HR, Walker JS, Rhee E, Kuller JA. Risk factors for birth defects. Obstet Gynecol Surv. 2017 Feb;72(2):12335.

4. Oliveira-Brancati $\mathrm{Cl}$, Ferrarese VC, Costa AR, Fett-Conte AC. Birth defects in Brazil: outcomes of a population-based study. Genet Mol Biol. 2020 Feb;43(1):e20180186.

5. Guimarães AL, Barbosa CC, Oliveira CM, Maia LT, Bonfim CV. Relationship of databases of live births and infant deaths for analysis of congenital malformations. Rev Bras Saúde Mater Infant. 2019;19(4):917-24.

6. Thomas EG, Higgins C, Westgate MN, Lin AE, Anderka M, Holmes LB. Malformations surveillance: comparison between findings at birth and age one year. Birth Defects Res. 2018 Jan;110(2):142-7.
7. Lin H, Luo MY, Luo JY, Zeng R, Li YM, Du QY, et al. Demographic Characteristics and Environmental Risk Factors Exposure of Birth Defects in Pregnant Women: A Population-based Study. Biomed Environ Sci. 2019 Jan;32(1):51-7.

8. Abreu MH, Lee KH, Luquetti DV, Starr JR. Temporal trend in the reported birth prevalence of cleft lip and/or cleft palate in Brazil, 2000 to 2013. Birth Defects Res A Clin Mol Teratol. 2016 Sep;106(9):789-92.

9. Almeida LF, Araujo Júnior E, Crott GC, Okido MM, Berezowski AT, Duarte $G$, et al. Epidemiological risk factors and perinatal outcomes of congenital anomalies. Rev Bras Ginecol Obstet. 2016 Jul;38(7):34855.

10. Cosme HW, Lima LS, Barbosa LG. Prevalence of congenital anomalies and their associated factors in newborns in the city of São Paulo from 2010 to 2014. Rev Paul Pediatr. 2017 Jan-Mar;35(1):33-8.

11. Luz GD, Karam SM, Dumith SC. Congenital anomalies in Rio Grande do Sul State: a time series analysis. Rev Bras Epidemiol. 2019;22:e190040.

12. Dutra LS, Ferreira AP. Association between birth defects and the use of agrochemicals in monocultures in the state of Paraná, Brazil. Saúde debate. 2017; 41(spe2):241-253.

13. Albuquerque MF, Souza WV, Araújo TV, Braga MC, Miranda Filho DB, Ximenes RA, et al. The microcephaly epidemic and Zika virus: building knowledge in epidemiology. Cad Saude Publica. 2018 Oct;34(10):e00069018.

14. Vilaça CR, Martins RC. Descriptive analysis of newborns with congenital malformation in Pernambuco. VEREDAS. 2017;109(1):59-68.

15. Mendonça JG, Guimarães MJB, Mendonça VG, Portugal JL, Mendonça CG. Profile of hospitalizations in Pediatric Intensive Care Units of the Brazilian Unified Health System in the state of Pernambuco, Brazil. Ciênc saúde coletiva. 2019;24(3):907-916.

16. Szwarcwald CL, Leal MD, Esteves-Pereira AP, Almeida WD, Frias PG, Damacena GN, et al. [Evaluation of data from the Brazilian Information System on Live Births (SINASC)]. Cad Saude Publica. 2019 Oct;35(10):e00214918.

17. Brasil. Secretaria de Vigilância em Saúde. Departamento de Análise de Situação de Saúde. Manual de instruções para o preenchimento da declaração de nascido vivo. 4th ed. Brasília: Ministério da Saúde; 2011.

18. Instituto Brasileiro de Geografia e Estatística. Cidades [Internet]. 2016 [citado em 2016 ago 28]. Disponível em: <https://www.ibge.gov.br/ cidades-e-estados/pe/recife.html? >

19. Oliveira MM, Andrade SS, Dimech GS, et al. Evaluation of the National Information System on Live Births in Brazil, 2006-2010. Epidemiol Serv Saude. 2015;24(4):629-40.

20. Maia LT, Souza WV, Mendes AD, Silva AG. Use of linkage to improve the completeness of the SIM and SINASC in the Brazilian capitals. Rev Saude Publica. 2017 Dec;51:112.

21. Frias PG, Szwarcwald CL, Lira PI. [Evaluation of information systems on live births and mortality in Brazil in the 2000s]. Cad Saude Publica. 2014 0ct;30(10):2068-280.

22. Groisman B, Mastroiacovo P, Barbero P, Bidondo MP, Liascovich R, Botto LD. A proposal for the systematic assessment of data quality indicators in birth defects surveillance. Birth Defects Res. 2019 Apr;111(6):324-32.

23. Pinto EP Junior, Luz LA, Guimarães MA, Tavares LT, Brito TR, Souza GF. Prevalence and associated factors of congenital abnormalities in newborns. Rev Bras Promoç Saúde. 2017;30(3):1-9. 
24. Lima NA, Silva CF, Santos, MM, Fernandes TAAM, Lucena EES. The epidemiological profile of congenital malformations in newborns from Rio Grande do Norte State from 2004 to 2011. 2018, R bras ci Saúde 22(1): 45-50.

25. de Araújo TV, Rodrigues LC, de Alencar Ximenes RA, de Barros MirandaFilho D, Montarroyos UR, de Melo AP, et al.; investigators from the Microcephaly Epidemic Research Group; Brazilian Ministry of Health; Pan American Health Organization; Instituto de Medicina Integral Professor Fernando Figueira; State Health Department of Pernambuco. Association between Zika virus infection and microcephaly in Brazil, January to May, 2016: preliminary report of a case-control study. Lancet Infect Dis. 2016;16(12):1356-63.

26. Vargas A, Saad E, Dimech GS, Santos RH, Sivini MA, Albuquerque $\mathrm{LC}$, et al. Characteristics of the first cases of microcephaly possibly related to Zika virus reported in the Metropolitan Region of Recife, Pernambuco State, Brazil. Epidemiol Serv Saude. 2016;25(4):691700.

27. Marinho F, Araújo VE, Porto DL, Ferreira HL, Coelho MR, Lecca RC, et al. Microcephaly in Brazil: prevalence and characterization of cases from the Information System on Live Births (Sinasc), 2000-2015. Epidemiol Serv Saude. 2016;25(4):701-12.

28. Toufaily MH, Westgate MN, Lin AE, Holmes LB. Causes of Congenital Malformations. Birth Defects Res. 2018;110(2):87-91.
29. Qadir M. Amir Shahril, Bano S. Prevalence and associated risk factors of congenital anomalies at a tertiary care hospital. P J M H S. 2017;11(3):942-4.

30. Mekonen HK, Nigatu B, Lamers WH. Birth weight by gestational age and congenital malformations in Northern Ethiopia. BMC Pregnancy Childbirth. 2015;15(1):76.

31. Nhoncanse GC, Germano CM, de Avó LR, Melo DG. Maternal and perinatal aspects of birth defects: a case-control study. Rev Paul Pediatr. 2014;32(1):24-31.

32. Schummers L, Hutcheon JA, Hacker MR, VanderWeele TJ, Williams PL, McElrath TF, et al. Absolute risks of obstetric outcomes by maternal age at first birth: a population-based cohort. Epidemiology. 2018;29(3):379-87.

33. Afroze S, Mannan M, Dey S, Moni S, Shabuj MK, Jahan I, et al. Risk Factors and Complications of Newborns with Birth Defect: A Hospital based CaseControl Study. Bangladesh Journal of Medical Science. 2019;19(1):133-40.

34. Cecile NM, waKumwimba M, Romain NS, Dressen I, Hugues L, Pascal $\mathrm{K}$, et al. Clinically visible congenital birth defects, case of the Kenya Health District in the Democratic Republic of the Congo. Open Access Library Journal. 2019;6(05):e5440.

35. Rodrigues LS, Lima RH, Costa LC, Batista RF. Characteristics of children born with congenital malformations in São Luís, Maranhão, Brazil, 2002-2011. Epidemiol Serv Saude. 2014;23(2):295-304. 